\title{
DIFERENCIAS NEUROPSICOLÓGICAS, BIOGRÁFICAS, PSICOLÓGICAS, FAMILIARES Y COMUNITARIAS, ENTRE ADOLESCENTES CON Y SIN TRASTORNO DISOCIAL*
}

\section{NEUROPSYCHOLOGICAL, BIOGRAPHIC, PSYCHOLOGICAL, FAMILIAR, AND COMMUNITIES' DIFFERENCES BETWEEN FEMALE AND MALE ADOLESCENTS WITH AND WITHOUT CONDUCT DISORDER}

\author{
César Armando ReY-Anacona ${ }^{* *}$, LIZZet Cristina Paitán-Ávila ${ }^{* * *}$ Y \\ ZULMA LORENA MONGUí-MERCHÁN****
}

\author{
*Trabajo financiado por la Dirección de Investigaciones de la Universidad Pedagógica y Tecnológica de \\ Colombia (Código SGI-582). \\ "Psicólogo y Doctor en Psicología Clínica y de la Salud. Profesor de Planta de la Universidad Pedagógica y \\ Tecnológica de Colombia y Director del Grupo de Investigación en Psicología Clínica y de la Salud. \\ E-Mail: cesar.rey@uptc.edu.co \\ Calle 24 No. 5-63, Antiguo Hospital San Rafael.Tunja - Colombia. \\ ***Psicóloga. Miembro del Grupo de Investigación en Psicología Clínica y de la Salud. \\ ***t* Psicóloga. Miembro del Grupo de Investigación en Psicología Clínica y de la Salud.
}

RESUMEN

El objetivo del estudio que se informa fue comparar un grupo de adolescentes de ambos sexos que cumplían los criterios diagnósticos de trastorno disocial (TD), entre sí y con respecto a un grupo de adolescentes de ambos sexos sin TD, en un conjunto de variables neuropsicológicas, biográficas, psicológicas / conductuales, familiares y comunitarias. Asimismo, se comparó la frecuencia general y específica de las conductas disociales reportadas por los participantes con TD. Todos los participantes tenían entre 11 y 18 años de edad. Los adolescentes con TD (60 varones y 11 mujeres) fueron seleccionados en tres instituciones reeducativas de libertad vigilada, mientras que los adolescentes sin TD (74 varones y 25 mujeres), en un centro de capacitación laboral para adolescentes de escasos recursos. Las mujeres con TD reportaron castigos físicos severos más frecuentemente que los varones con TD, aunque a una edad mayor que éstos, presentando mayores puntuaciones en Neuroticismo y menores en Retroalimentación personal, así como una frecuencia mayor de conductas disociales. Las comparaciones por sexo revelaron que tanto los varones como las mujeres con TD, comparados con los adolescentes sin TD, presentaron puntuaciones más altas en Extroversión y un mayor consumo de sustancias psicoactivas, cambios y expulsiones escolares, escapadas del hogar, pensamientos suicidas y una menor edad de consumo de marihuana. Sin embargo, las mujeres con TD evidenciaron puntuaciones mayores en Machismo y menores en Empatía, Fluidez fonémica y Retroalimentación personal. Estos resultados sugieren más dificultades entre las adolescentes con TD que deberían considerarse en la evaluación y el tratamiento.

Palabras clave: Trastorno disocial; Adolescentes; Diferencias por sexo; Factores de riesgo; Conducta antisocial. 


\section{ABSTRACT}

Gender differences in risk behaviors for health, risk factors and symptoms of conduct disorder (CD) have not been sufficiently investigated, although knowledge of these differences help define more effective strategies for assessment, prevention and treatment. The aim of this study was to compare a group of male and female adolescents who met the diagnostic criteria of $\mathrm{CD}$, among themselves and with respect to a group of male and female adolescents without CD, on a set of neuropsychological, biographical, psychological / behavioral, family and community variables identified in the specialized literature. Also, the general and specific frequency of antisocial behavior was compared between participants with CD. All participants were between 11 and 18 years old, with a mean of 16 years, and living in low socioeconomic neighborhoods. Adolescents with $\mathrm{CD}, 60$ males and 11 females, were selected in three re-educational institutions of probation, while adolescents without $\mathrm{CD}, 74$ males and 25 females, were selected in a job training center for low-income teenagers. The two groups did not differ significantly in age, sex and socioeconomic status. The instruments used were: Color-word Test (Golden, 1994), Neuropsychological Assessment of Children (Matute, Rosselli, Ardila, \& Ostrosky-Solís, 2007), Self-Report Questionnaire of Partner Violence Incidents (Rey-Anacona, 2008), Spanish Version of Dyadic Adjustment Scale (Echeburúa \& de Corral, 1998), Depression Inventory (Beck, Rush, Shaw, \& Emery, 1983), Masculinity and Femininity Inventory (Lara, 1993), Scale of Empathy (Martorell, González \& Calvo, 1998), Eysenck Personality Inventory (Eysenck, H.J. \& Eysenck, S.G.B., 1984), Questionnaire of Child and Adolescent Self-Control (Capafons \& Silva, 2001), Brief Ques- tionnaire for Diagnosing Attention Deficit (Pineda et al., 1999) and two questionnaires developed in the research, reviewed by judges on methodology and content. An observational, descriptive, comparative, cross-sectional design was used, involving both adolescents and their mothers, implemented the following statistical tests: (a) t-test for two independent samples and effect size (Cohen's $d$ ) for interval and ratio variables, (b) Mann Whitney $U$ for ordinal variables, and (c) $J i$ Square and Odds
Ratio for nominal variables. Women with CD reported severe physical punishment more frequently than men with $\mathrm{CD}$, although at an older age, and they showed higher scores on Neuroticism, and lower on Personal Feedback, as well as a higher frequency of antisocial behaviors, both aggressive and covert, and difficulties at work as a result of such conducts. Comparisons by sex revealed both men and women with $\mathrm{CD}$, compared to adolescents without $\mathrm{CD}$, had higher scores on Extraversion, and increased consumption of psychoactive substances, school changes and expulsions, runaways, suicidal thoughts and younger of marijuana. However, women with CD showed higher scores on Machismo, and minor in Empathy, Personal Feedback, and Phonemic Fluency than women without $\mathrm{CD}$, while the mothers of men with $\mathrm{CD}$ reported most frequently gangs in their place of residence. These results suggest that women with $C D$ may have a higher frequency of antisocial behaviors and antisocial profiles similar or more severe than male adolescents with $\mathrm{CD}$, and both males and females have a high frequency of conditions and behaviors that undermine their physical and mental health, which may worsen prognosis, aspects that should be considered in the evaluation and treatment. They also suggest that greater importance should be provided to treatment alternatives that minimize the effect of multiple risk behaviors for physical and mental health that demonstrate these adolescents.

Key words: Conduct disorder; Teenagers; Sex differences; Risk factors; Antisocial behavior.

\section{INTRODUCCIÓN}

El trastorno disocial (TD) se define como un patrón de comportamiento caracterizado por la realización repetitiva y persistente de actos en contra de las normas sociales y los derechos de los demás y se inicia generalmente en la infancia o la adolescencia. Son actos que incluyen agresiones a personas o animales, la destrucción de la propiedad pri- 
vada, la fraudulencia o robo y las violaciones graves de las normas del grupo de referencia, de los cuales al menos tres se han presentado en el último año, generando un deterioro significativo en la vida académica, escolar y/o laboral del individuo (American Psychiatric Association, 2013).

Los estudios sobre las características relacionadas con el TD revelan que en su desarrollo podrían incidir variables neurobiológicas, biográficas, psicológicas, familiares y comunitarias, reportándose a nivel neurobiológico una mayor prevalencia de signos neurológicos menores, más antecedentes negativos a nivel pre y perinatal, indicadores de un funcionamiento autónomo deficitario y puntuaciones significativamente más bajas en pruebas de funciones ejecutivas, especialmente en fluidez verbal, memoria de trabajo y razonamiento espacial (Fairchild et al., 2008; Harrington \& Maskey, 2008; Toupin, Dery, Pauze, Mercier \& Fortin, 2003).

El TD también se ha relacionado con un temperamento difícil en los primeros años de vida (American Psychiatric Association, 2013; Schmeck \& Poutstka, 2001), malos tratos físicos y sexuales (Baker, 2008), rasgos psicológicos tales como impulsividad, búsqueda de sensaciones, inconformismo y extroversión (e.g., Daderman, 1998), síntomas y comorbilidad con el trastorno por déficit de atención con hiperactividad (TDAH - American Psychiatric Association, 2013; e.g., Toupin et al., 2003), puntajes más bajos en empatía (e.g., Rey-Anacona, 2003), puntuaciones más bajas en pruebas de habilidades verbales (e.g., Toupin et al., 2003) y observación de violencia en medios masivos de comunicación (e.g., Moeller-Nehring, Moach, Castell, Weigel \& Meyer, 1998). Asimismo, este trastorno ha sido relacionado con una crianza autoritaria y mayor castigo corporal (e.g., Frick, Christian \& Wooton, 1999; Toupin et al., 2003), falta de supervisión paterna (e.g., Frick et al., 1999), inconsistencia en la aplicación de disciplina (Baker, 2008; Harrington \& Maskey, 2008), depresión materna y paterna (Ohannessian et al., 2005) y circunstancias familiares adversas como bajos ingresos económicos, alcoholismo paterno y observación de violencia doméstica
(Baker, 2008; Evans, Davis \& DiLillo, 2008; Toupin et al., 2003). La conducta antisocial en la adolescencia, particularmente, ha sido relacionada con circunstancias socio-familiares como antecedentes de conducta criminal en los padres, alcoholismo paterno, un número alto de hermanos, juventud de la madre, familia de padre único o reconstituida, ruptura y discordia marital, vecindad peligrosa, pares negativos y bajo nivel socioeconómico (Rutter, Giller \& Hagell, 2000).

Los niños y adolescentes de ambos sexos con TD tienden a presentar problemas escolares, fracaso académico y otras dificultades que pueden afectar su salud física y mental, tales como un inicio más temprano en el consumo de bebidas alcohólicas y sustancias ilegales, trastornos por uso de sustancias, problemas legales, malas relaciones con pares y adultos, promiscuidad sexual, embarazos no deseados e intentos suicidas (American Psychiatric Association, 2013; Dicker, Morrissey, Abikoff \& Alvir, 1997; Fairchild et al., 2013; Harrington \& Maskey, 2008).

Las diferencias por sexo en relación con los factores de riesgo y las características del TD constituyen una línea de investigación que se inició buscando explicar las diferencias que existen en la prevalencia de dicho trastorno (Eme \& Kavanaugh, 1995), la cual es mucho mayor entre los varones que entre las mujeres (American Psychiatric Association, 2013; Baker, 2008; Berkout, Young \& Gross, 2011; Harrington \& Maskey, 2008).

La edad de inicio del TD también tiende a ser menor en los varones (American Psychiatric Association, 2013; Keenan, Loeber \& Green, 1999; Masi et al., 2007) y algunas investigaciones indican diferencias por sexo en la comorbilidad del TD que señalan en general, que las mujeres con dicho trastorno tienen una mayor probabilidad de presentar trastornos internalizantes, como los afectivos o de ansiedad, mientras que los varones tienden a presentar trastornos de tipo externalizante (Berkout et al., 2011; Keenan et al., 1999). Masi y colaboradores (2007) informaron una proporción mayor de TDAH entre los varones que entre las mujeres, en un grupo de menores de edad remitidos a tratamiento. 
Whitmore y colaboradores (1997), por su parte, encontraron en un grupo de adolescentes remitidos por TD, que el trastorno depresivo mayor era más común en las mujeres, mientras que en los varones era más frecuente la dependencia a sustancias. Lehto-Salo, Närhi, Ahonen y Marttunen (2009) compararon 40 mujeres entre 12 y 18 años que tenían TD o trastorno negativista desafiante (TND), con 37 adolescentes varones con los mismos trastornos, encontrando en las primeras una mayor comorbilidad con el trastorno depresivo mayor, trastornos de ansiedad y trastornos por uso de sustancias, mientras que el trastorno de la lectura era más común en los varones.

Estas diferencias en la prevalencia, la edad de inicio y la comorbilidad del TD por sexo indican que los factores de riesgo de este trastorno podrían diferir entre varones y mujeres. Sin embargo, la mayoría de las investigaciones sobre este tópico se han centrado históricamente en los varones con TD o con problemas de comportamiento (Berkout et al., 2011; Lehto-Salo et al., 2009; Pager, Leininger \& Gardner, 2010). La revisión realizada por Berkout y colaboradores (2011), con artículos publicados entre 2000 y 2011, señala que los factores de riesgo de tipo socio-familiar como los patrones de crianza disfuncionales y la pobreza, son comunes tanto en varones como mujeres con TD. No obstante, otros trabajos indican diferencias en este tipo de factores de riesgo. Ilomäki y colaboradores (2006), por ejemplo, encontraron en 278 adolescentes varones y mujeres con TD, vinculados a una institución psiquiátrica, que el riesgo de presentar este trastorno era significativamente mayor en las adolescentes que habían sido maltratadas físicamente y que provenían de familias disfuncionalesl, mientras que Lehto-Salo y colaboradores (2009) hallaron más adversidades familiares en mujeres con TD que en varones con dicho trastorno.

Según una revisión efectuada por Keenan y colaboradores (1999) y otra posteriormente por Berkout y colaboradores (2011), las mujeres tendrían más rasgos de personalidad predisponentes de TD que los varones. Sin embargo, Taylor e Iacono (2007) encontraron puntuaciones más bajas en rasgos de personalidad relacionados con el seguimiento de reglas sociales y autoridad, autocontrol y evitación, en adolescentes varones y mujeres con TD comparados con controles, pero no hallaron diferencias por sexo en estos rasgos. Otros investigadores realizaron comparaciones solamente con mujeres, hallando en general resultados similares a los encontrados entre los varones con TD. Haggerty, Nevid y Moulton (1998), por ejemplo, compararon 36 adolescentes mujeres con TD y TND, con 40 adolescentes normales, encontrando entre las primeras niveles más altos de ansiedad y un menor desempeño en fluidez verbal, aunque no encontraron diferencias en la resolución cognitiva de problemas interpersonales. Lewis (2010) halló una mayor prevalencia vida de TD y su progresión hacia el trastorno de personalidad antisocial y los trastornos por sustancias, en un grupo de mujeres adultas encarceladas, lo que indica que las mujeres con TD también tendrían un alto riesgo de presentar estos dos trastornos en la vida adulta, como los varones con dicho desorden (Keenan et al., 1999; Fairchild et al., 2013).

Según Eme (2007), la mayor prevalencia del TD en los varones se debería a la influencia de factores biológicos, como altos niveles de testosterona y factores socioculturales, como las expectativas de género que promueven la agresividad en los varones, predisponiéndolos desde los años preescolares a dicho trastorno. Existe evidencia que muestra que los niños son más agresivos que las niñas desde los 17 meses de edad (Berkout et al., 2011), lo que señala una mayor predisposición biológica hacia el TD entre ellos. No obstante, se han informado niveles más bajos de cortisol tanto en adolescentes varones con TD (Fairchild et al., 2008), como en adolescentes mujeres con el mismo trastorno (Pager et al., 2006), así como un volumen de la amígdala derecha más pequeño en adolescentes varones y mujeres con TD, comparados con controles sanos (Fairchild et al., 2013), lo que sugiere que los factores biológicos predisponentes del TD no son exclusivos de éstos. 
En relación con las diferencias por sexo en los tipos y la severidad de las conductas disociales también se han desarrollado muy pocas investigaciones, aunque la American Psychiatric Association (2013) señala que los varones cometen más agresiones directas que las mujeres, quienes tienden a presentar más actos antisociales furtivos, como mentiras, consumo de sustancias y escapadas de casa. No obstante, Lehto-Salo y colaboradores (2009) no encontraron diferencias en el número de síntomas de TD entre adolescentes varones y mujeres con dicho trastorno y con TND, aunque no examinaron las diferencias específicas en este tipo de síntomas.

La literatura sobre las diferencias por sexo en las condiciones y comportamientos que afectan la salud física y mental de los adolescentes con TD también es escasa. Whitmore y colaboradores (1997) informaron una alta frecuencia de conductas sexuales de riesgo en adolescentes mujeres que presentaban TD y dependencia o abuso de sustancias, pero no compararon este dato con un grupo de controles o con varones con TD. Pager, Kazmi, Gardner y Wang (2007) efectuaron un estudio longitudinal con 52 adolescentes con TD comparadas con 41 controles, presentando las primeras una peor salud general al inicio de su vida adulta, más baja autoestima, menos hábitos y conductas saludables y más embarazos a temprana edad. Lehto-Salo y colaboradores (2009), por su parte, hallaron un mayor riesgo suicida entre las mujeres que entre los varones en su ya citado estudio, lo cual señala diferencias importantes a nivel de estas conductas de riesgo.

En resumen, las diferencias por sexo en las conductas de riesgo para la salud, los factores de riesgo y la sintomatología del TD no han sido suficientemente investigados, a pesar de que este trastorno ha sido relacionado con factores de diversa índole y el conocimiento de estas diferencias ayudaría a delimitar estrategias de evaluación, prevención y tratamiento más eficaces. El estudio de las diferencias según sexo en relación con los diferentes trastornos psicopatológicos, ha sido resaltado como una de las principales vías para incrementar el conocimiento sobre su etio- logía (Raine, Yang, Narr \& Toga, 2011). En Colombia, en particular, tiende a ser escaso el conocimiento sobre las características y síntomas del TD, y sus diferencias por sexo, aunque Posada-Villa, Aguilar-Gaxiola, Magaña y Gómez (2004) informaron que la prevalencia durante toda la vida de dicho trastorno alcanzaba el $8.8 \%$ de los varones y el $2.7 \%$ de las mujeres, en una muestra nacional de 4.544 individuos de 18 a 65 años.

Así, con el fin de subsanar los vacíos referentes a la falta de comparación entre varones y mujeres con TD y con individuos sin dicho trastorno, e incrementar el conocimiento sobre el tema, el estudio que se informa tuvo como objetivo comparar un grupo de adolescentes varones y mujeres con TD entre sí y también con un grupo de adolescentes de ambos sexos sin TD con rasgos sociodemográficos similares, en relación con un amplio número de variables neuropsicológicas, biográficas, psicológicas / conductuales, familiares y comunitarias, señaladas en la literatura especializada, previamente citada y comparar la frecuencia general y específica de las conductas disociales reportadas por los participantes con TD.

\section{MÉTOdO \\ PARTICIPANTES}

Los participantes fueron 170 adolescentes de 11 a 18 años $(M=16 ; D T=1.33)$, que vivían en barrios de estrato socioeconómico bajo-medio $(51.8 \%)$, medio-bajo $(30 \%)$ y bajo-bajo (14.7\%), según la clasificación del Departamento Nacional de Estadística de Colombia. De ellos, 60 varones y 11 mujeres cumplían los criterios diagnósticos del TD de la American Psychiatric Association (2013) y se encontraban en tres instituciones de reeducación de Bogotá, que atienden menores infractores de la ley bajo la medida de libertad vigilada. Se seleccionaron estas instituciones debido a que en ellas se encuentra una alta prevalencia de TD (e.g., Rioseco et al., 2009 , encontraron una prevalencia de $46.99 \%$ entre 100 adolescentes infractores institucionalizados). 
Para verificar el cumplimiento de los criterios diagnósticos de la American Psychiatric Association, dichos participantes debían informar la realización en el último año de por lo menos tres de las conductas disociales que señala dicha organización, presentes en el Cuestionario Breve para el Diagnóstico del Trastorno Disocial (Pineda, Puerta, Arango, Calad \& Villa, 2000) y que dichas conductas hubieran generado deterioro en el área educativa, laboral y/o social, ocasionando dificultades en la escuela o el colegio, el trabajo o con familiares, amigos u otras personas, aspectos que fueron corroborados a través de los informes que tenían en las instituciones donde fueron contactados.

Este grupo fue comparado con 99 adolescentes (74 varones y 25 mujeres) que no cumplían los criterios diagnósticos de TD y que fueron contactados en una institución de capacitación laboral para menores de escasos recursos económicos de Bogotá. Se seleccionaron participantes de esta institución por las siguientes razones: (a) la probabilidad de cumplir los criterios diagnósticos de TD con los usuarios de esta institución era más baja que con los usuarios de las instituciones de reeducación, lo que permitió conformar el grupo de comparación con un bajo margen de error y (b) dichos participantes provenían de barrios de Bogotá pertenecientes a los mismos estratos socioeconómicos de los participantes con TD y tenían el mismo rango de edades. Estos adolescentes no informaron las conductas disociales presentes en el Cuestionario Breve para el Diagnóstico del Trastorno Disocial (Pineda et al., 2000), aspecto que fue corroborado con el informe del equipo interdisciplinario de la institución.

Las dos grupos de participantes fueron vinculados a la investigación según su disponibilidad y no diferían en edad $[t(170)=-1.584$, $p=.115$, bilateral; $d=-.244]$, sexo $\left[\chi^{2}(1\right.$, $170)=2.359, p=.133$, bilateral $]$ y estrato socioeconómico $[U(N=170)=3506.0, p=.976$, bilateral].

\section{INSTRUMENTOS}

En la Tabla 1 se informan las variables estudiadas y sus correspondientes instrumentos administrados, los cuales se describen a continuación:

1.- Cuestionario Breve para el Diagnóstico del TRAstorno Disocial (PIneda et al., 2000)

Es un cuestionario de auto-informe que contiene los síntomas del Criterio A para el diagnóstico del TD de la American Psychiatric Association (2013) -excepto el referente a las escapadas de la escuela-, desarrollado y validado con una muestra de 190 adolescentes varones colombianos entre 12 y 16 años. El instrumento evidenció un buen nivel de consistencia interna $(\alpha=.86)$ y tres factores estables para 11 ítemes, que explicaron el 53.9\% de su variancia.

\section{2.- Test de Colores y Palabras (Golden, 1994)}

Es una prueba neuropsicológica ampliamente utilizada en la práctica clínica y la investigación, compuesta por tres subpruebas de 45 segundos cada una, la última de las cuales mide la capacidad de inhibición de una respuesta automática en favor de una tarea controlada, considerada una función ejecutiva (Pineda, 2000). El autor informó índices de correlación test-retest de $.89, .84$ y .73 para las tres subpruebas, y la subprueba de interferencia correlacionó significativamente con medidas similares.

3.- EVALUACIÓN NEUROPSICOLÓGICA INFANTIL (ENI Matute, Roselli, Ardila \& Ostrosky-Solís, 2007)

Es una batería neuropsicológica dirigida a niños y adolescentes hispanohablantes que explora 14 áreas cognitivas diferentes. Sus datos normativos se obtuvieron con una muestra aleatoria de niños y adolescentes de ambos sexos escolarizados de Colombia y México, presentando datos adecuados en pruebas de confiabilidad test-retest (.86) e inter-calificadores (.99) y correlaciones estadísticamente significativas con escalas similares del WISCR. 
En esta investigación se utilizaron las tres pruebas de fluidez verbal del instrumento (dos de fluidez semántica y una de fluidez fonémica), la cual se considera una función ejecutiva ya que tiene que ver con la producción verbal controlada (Marino, Acosta Mesas \& Zorza, 2011; Pineda, 2000).

4.- Cuestionario de Autolnforme sobre INCIdentes de Violencia Conyugal (Rey-Anacona, 2008)

Este cuestionario permite informar el atestiguamiento de 14 conductas de violencia realizadas por parte del padre hacia la madre y las mismas 14 de la madre hacia el padre, para un total de 28 ítemes de tipo Sí-No. Como formas de violencia se contemplan la física y verbal, amenazas, encerramientos, prohibiciones, echar de la casa a la otra persona, violencia sexual, violencia económica y amenazas a personas cercanas. El instrumento fue validado a nivel metodológico y de constructo por jueces expertos, evidenciando un buen nivel de consistencia interna $(\alpha=.86)$.

5.- VERSIÓN ESPAÑOLA (ECHEBURÚA \& DE CORRAL, 1998) DE LA ESCALA DE AJUSTE DIÁdICO (SPANIER, 1976)

Este instrumento examina el grado de acuerdo entre los cónyuges en 15 áreas de la vida en pareja, la frecuencia con la que se presentan 13 situaciones funcionales y disfuncionales, el grado de felicidad generado por la relación y el futuro de la misma. Sus 32 ítemes conforman las siguientes subescalas: Satisfacción diádica, Cohesión, Consenso y Expresión de afecto. En esta investigación el Alpha de Cronbach fue igual a .97.

6. - INVENTARIO DE DEPRESIÓN (IDB - BECK, RUSH, SHAW \& EMERY, 1983)

Es un instrumento muy utilizado en la práctica y en la investigación, cuyos ítemes contienen los principales síntomas de la depresión clínica. En esta investigación el Alpha de Cronbach fue igual a .89.

\section{7.- INVENTARIO dE MASCULINIDAd y FEMINIDAD (IMAFE - LARA, 1993)}

Esta prueba permite la auto-descripción de la personalidad a través de 60 adjetivos referentes a cuatro rasgos de personalidad de género: Masculinidad, Femineidad, Machismo y Sumisión, los cuales conforman cuatro escalas con quince reactivos tipo Likert. La Escala de Machismo permite autoevaluar rasgos relacionados con la agresividad, la dominación y la intransigencia.

En Colombia se evaluó su confiabilidad y validez de constructo con 1.527 varones y mujeres entre 15 y 42 años, presentando sus escalas los siguientes valores Alpha: Masculinidad: .78, Femineidad: .88, Machismo: .82 y Sumisión: .79. El análisis factorial arrojó tres factores que explicaban el $30.8 \%$ de la variancia (Martínez-Gómez, Guerrero-Rodríguez \& Rey-Anacona, 2012).

\section{8.- Escala de Empatía (Rey-Anacona, 2003)}

Contiene 15 ítemes tipo Likert del Cuestionario de Conducta Prosocial de Martorell, González y Calvo (1998) y fue validada con 318 preadolescentes y adolescentes colombianos de 11 a 18 años de edad. El instrumento evidenció una buena consistencia interna $(\alpha=.78)$ y discriminó significativamente individuos con TD y $\sin$ TD $(p=.008)$.

\section{9.- Cuestionario de Personalidad de Eysenck (EPI - EYSENCK H.J. \& EYSENCK, S.G.B, 1998)}

Permite ponderar en adolescentes y adultos dos dimensiones opuestas de la personalidad: Neuroticismo - Estabilidad y Extroversión - Introversión, y han sido confirmadas por múltiples análisis factoriales y correlacionales. Su confiabilidad test-retest y su consistencia interna es superior a .80 (Eysenck, 
H. \& Eysenck, S., 1998), presentando en esta investigación un Alpha igual a .82.

10.- Cuestionario de Autocontrol INFANTIL Y AdolesCENTE (CACIA - CAPAFÓNS \& SILVA, 2001)

Este cuestionario evalúa los procesos básicos y habilidades necesarias para que se produzca un comportamiento auto-controlado, considerando tres dimensiones positivas (Retroalimentación personal, Retraso de la recompensa y Autocontrol criterial) y una dimensión negativa (Autocontrol procesual). La consistencia interna de sus escalas osciló entre .50 y .79 y estas correlacionaron positiva o negativamente con medidas teóricamente relacionadas (Capafóns \& Silva, 2001), presentando en esta investigación un Alpha de Cronbach igual a .80 .

11.- Cuestionario Breve para el Diagnóstico de Deficiencia Atencional (PIneda et al., 1999)

Permite reportar la presencia de síntomas referentes a los tres subtipos del TDAH, estipulados en el Criterio A de la American Psychiatric Association (2013), por parte de la madre del menor y en base a 18 ítemes tipo Likert de cuatro opciones. Conforme a la American Psychiatric Association, se considera que el individuo podría presentar TDAH si informa al menos seis síntomas de alguno de los dos subtipos básicos (inatento e hiperactivo - impulsivo) o el subtipo combinado si informa al menos seis síntomas de los dos. Pineda y colaboradores reportaron niveles adecuados de consistencia interna obtenidos con una muestra de 540 niños y adolescentes colombianos de ambos sexos, con un Apha de Cronbach igual a .92 para los ítemes de inatención y .78 para los de hiperactividad - impulsividad, y una estructura estable de dos dimensiones que explicaban el $36.9 \%$ y el $14.1 \%$ de la variancia, agrupando los ítemes de inatención e hiperactividad - impulsividad, respectivamente, coherentes con los dos principales subtipos de TDAH descritos por la American Psychiatric Association.
12.- Cuestionario de Autoinforme de VariaBLES PSICOLÓGICAS

Se desarrolló en esta investigación para recoger información por parte de los adolescentes sobre las variables biográficas y psicológicas señaladas en la Tabla 1 , conforme a 49 ítemes con diferentes opciones de respuesta. Para obtener información sobre el consumo de alcohol y otras sustancias psicoactivas, la conducta sexual de riesgo y la ideación suicida e intentos de suicidio, se incluyeron y adaptaron varios ítemes del Youth Risk Behavior Surveillance System (YRBSS - Brener et al., 2004), mientras que para los ítemes referentes a la historia de abuso físico y sexual, se tuvieron en cuenta los reactivos de historia de violencia familiar desarrollados por Renner y Slack (2006). El instrumento fue revisado a nivel metodológico y de contenido por expertos y fue depurado con una muestra piloto de 20 adolescentes de ambos sexos.

13.- Cuestionario de VARIABLES BIOGRÁFICAS, PSICOLÓGICAS, FAMILIARES Y COMUNITARIAS

Se desarrolló en esta investigación con el fin de obtener información sobre las variables biográficas, familiares y comunitarias señaladas en la Tabla 1, mediante su administración a las madres de los adolescentes. Contiene 46 ítemes con diferentes opciones de respuesta. Para la información concerniente al consumo de bebidas alcohólicas y otras sustancias psicoactivas por parte de los padres, también se realizó una adaptación de ítemes del YRBSS (Brener et al., 2004). El instrumento fue revisado a nivel metodológico y de contenido por expertos, siendo probado con las madres de los adolescentes que participaron en el estudio piloto del instrumento anterior.

\section{Procedimiento Y ANÁLISIS DE LOS DATOS}

El diseño utilizado fue observacional, descriptivo, comparativo y transversal. 
En las reuniones familiares que se realizaban en las instituciones con los participantes y sus madres se les solicitó su consentimiento suministrándoles información sobre el objetivo, el procedimiento, la confidencialidad y la independencia de la investigación, así como la voluntariedad de su participación, las posibles molestias que podía traer la aplicación de los instrumentos y el compromiso de dar los resultados de los instrumentos aplicados si lo deseaban.

La administración de los instrumentos fue grupal y se solicitó a las madres que respondieran los siguientes instrumentos para mejorar la confiabilidad de la información deseada: (a) Cuestionario de Variables Biográficas, Psicológicas, Familiares y Comunitarias, (b) Escala de Ajuste Diádico (Spanier, 1976), (c) IDB (Beck et al., 1983) y (d) Cuestionario Breve para el Diagnóstico de Deficiencia Atencional (Pineda et al., 1999). Los demás instrumentos fueron contestados por los adolescentes de ambos sexos.

La información obtenida fue incorporada a una base de datos del programa estadístico SPSS versión 20.0 y las comparaciones se efectuaron mediante las pruebas estadísticas: (a) $t$ de Student para dos muestras independientes y tamaño del efecto ( $d$ de Cohen), para las variables de intervalo y de razón, (b) $U$ de Mann Whitney para las variables ordinales, y (c) Ji-cuadrado y Odds Ratio (OR) para las variables nominales, aceptándose una significancia igual o inferior a .05 , bilateral. Para comparar la frecuencia general y de cada uno de los síntomas entre los varones y mujeres con TD, se codificaron las respuestas a cada síntoma de acuerdo al Criterio A de la American Psychiatric Association (2013), presentes en el cuestionario diagnóstico de Pineda y colaboradores (1999): 0: Nunca, 1: Algunas veces, 2: Frecuentemente y 3: Siempre, efectuándose las comparaciones con la $t$ de Student y calculándose también el tamaño del efecto $(d)$.

\section{Resultados}

Se presentan inicialmente los resultados obtenidos con las comparaciones entre los dos grupos de varones y mujeres (con y sin TD) y posteriormente los resultados de las comparaciones intra-grupo (solamente varones y mujeres con TD).

Los varones con TD, comparados con los varones sin TD, presentaron un promedio significativamente mayor de:

a.- cantidad de personas con las que han tenido relaciones sexuales: grupo con TD $[M=6.29, D T=9.69$; grupo sin TD: $M=3.28, D T=2.47 ; \quad t(101)=2.152$, $p=.036 ; d=.425]$,

b.- número de personas con las que han tenido relaciones en los últimos tres meses [grupo con TD: $M=2.06, D T=1.87$; grupo sin TD: $M=1.35, D T=.99 ; t(91)=2.301, p=.024$; $d=.474]$,

c.- puntuaciones en la prueba de fluidez semántica con animales [grupo con TD: $M=17.73, D T=3.59$; grupo sin TD: $M=15.52, D T=4.12 ; t(106)=2.935$, $p=.004 ; d=.571] \mathrm{y}$

d.- puntajes en la Escala de Extroversión de la EPI (Eysenck, H.J. \& Eysenck, S.G.B., 1998) [grupo con TD: $M=14.38, D T=3.47$; grupo sin TD: $M=13.10, D T=3.11$; $t(132)=2.224, p=.028 ; d=.388]$.

Sus madres, por su parte, presentaron una media de puntuaciones significativamente mayor en:

g.- la Subescala de Satisfacción Diádica de la Escala de Ajuste Diádico (Echeburúa \& de Corral, 1998) [grupo con TD: $M=25.02$, $D T=15.09$; grupo sin TD: $M=18.51$, $D T=14.74 ; t(125)=2.343, p=.021$; $d=.436] \mathrm{y}$

h.- la Escala de Consenso de la misma prueba [grupo con TD: $M=35.56, D T=22.37$; grupo sin TD: $M=24.15, D T=20.74$; $t(125)=3.152, p=.002 ; d=.528]$.

Las mujeres con TD, por su parte, comparadas con las adolescentes sin $\mathrm{TD}$, mostraron un promedio significativamente menor de:

a.- años que tuvieron la primera relación sexual [grupo con TD: $M=13.44, D T=1.13$; grupo sin TD: $M=15.44, D T=1.51$; $t(18)=-3.182, p=.006 ; d=-1.49]$,

b.- puntuación total en la Escala de Empatía (Rey-Anacona, 2003) [grupo con TD: $M=33.36, D T=3.64$; grupo $\sin \mathrm{TD}$ : 
$M=39.44, D T=7.78 ; \quad t(36)=-3.191$, $p=.003 ; d=-1.0]$;

c.- puntajes en la Prueba de Fluidez Fonémica [grupo con TD: $M=7.29, D T=3.04$; grupo sin TD: $M=11.80, D T=3.10 ; t(27)=-3.363$, $p=.007 ; d=-1.46]$,

d.- puntuaciones en la Escala de Retroalimentación Personal de la CACIA (Capafóns \& Silva, 2001) [grupo con TD: $M=7.40$, $D T=2.76$; grupo $\sin \mathrm{TD}: M=11.21$, $D T=3.89 ; \quad t(34)=-3.230, p=.004$; $d=-1.129]$ y un promedio significativamente mayor de puntajes en:

e.- la Escala de Extroversión de la EPI (Eysenck \& Eysenck, 1998) [grupo con TD: $M$ $=15.91, D T=2.51$; Grupo sin TD: $M=12.75$, $D T=2.74 ; t(35)=3.359, p=.003 ; d=1.202]$; f.- la Escala de Machismo del IMAFE (Lara, 1993) [grupo con TD: $M=4.07, D T=1.24$; grupo sin TD: $M=2.87, D T=.71$; $t(36)=2.996, p=.010 ; d=1.187]$.

Los varones con TD, comparados con los participantes sin TD, informaron un número mayor de veces que:

a.- consumieron tragos de alcohol en los últimos 30 días: $U(134)=1594.5, p=.003$,

b.- tomaron más de cinco tragos en los últimos 30 días: $U(133)=1624.0, p=.006$,

c.- consumieron marihuana: $U(134)=1444.0$, $p=.000$,

d.- consumieron marihuana en los últimos 30 días: $U(134)=1748.0, p=.012$, e.- consumieron cocaína: $U(134)=1908$, $p=.005$,

f.- utilizaron inhalantes: $U(134)=1624.5$, $p=.000$,

g.- se sintieron descuidados por el padre: $U$ $(132)=1767.0, p=.035$,

h.- se escaparon de la casa: $U(132)=1468.5$, $p=.001$,

i.- cambiaron de escuela: $U(132)=1469.0$, $p=.001$.

Asimismo, informaron una edad menor de inicio de consumo de marihuana: $U(134)=1744.0, p=.027$.

Las mujeres con TD, por su parte, comparadas con las adolescentes sin TD, reportaron un número mayor de veces que:

a.- consumieron marihuana: $U(36)=41.5$, $p=.000$, b.- utilizaron marihuana en los últimos 30 días: $U(36)=68.0, p=.001$,

d.- consumieron inhalantes: $U(36)=76.0$, $p=.005$,

c.- consumieron éxtasis: $U(36)=87.5$, $p=.002$,

d.- consumieron bazuco: $U(36)=94.0$, $p=.013$,

e,- fueron castigadas físicamente de forma violenta: $U(36)=63.0, p=.005$,

f.- se escaparon de la casa: $U(36)=78.5$, $p=.025 \mathrm{y}$

g.- cambiaron de escuela: $U(36)=61.0$, $p=.006$.

Igualmente, reportaron una edad menor de inicio de consumo de marihuana que las adolescentes sin TD: $U(36)=61.0, p=.003$.

Un porcentaje mayor de varones con TD informaron las siguientes situaciones, comparados con los varones sin TD:

a.- haber tenido relaciones sexuales con penetración: $\left.\chi\right|^{2}(1,133)=6.589, p=.012$; $O R=3.266$,

b.- sentimientos de tristeza o desesperanza: $\chi^{2}(1,133)=10.913, p=.002 ; O R=4.117$,

c.- pensamientos suicidas en los últimos 12 meses: $\chi^{2}(1,133)=4.376, p=.048$; $O R=3.450$,

d.- haber sido expulsado de algún plantel educativo por mal comportamiento: $\chi^{2}(1$, $132)=12.972, p=.000 ; O R=3.680$,

e.- actos sexuales forzados por una persona mayor: $\chi^{2}(1,133)=4.919, p=.046$; $O R=8.000$,

f.- por un amigo: $\chi^{2}(1,133)=5.018$, $p=.039 ; O R=.933$,

g.- por un familiar: $\chi^{2}(1,133)=5.018$, $p=.039 ; O R=.933$.

Asimismo, reportaron más frecuentemente haberse escapado de la casa $\left[\chi^{2}(1\right.$, $132)=15.621, p=.000 ; O R=4.221]$, específicamente por:

- mala conducta personal: $\chi^{2}(1,131)=6.427$, $p=.016 ; O R=3.073$,

- por conflictos con un familiar: $\chi^{2}(1$, $131)=6.610, p=.015 ; O R=4.340 \mathrm{y}$

- por las condiciones económicas de la familia: $\chi^{2}(1,131)=4.943, p=.045 ; O R=8.038$.

Sus madres informaron con mayor frecuencia: 
1.- haber fumado durante el embarazo: $\chi^{2}(1$, $105)=4.664, p=.037 ; O R=3.308$,

m.- consumir bebidas alcohólicas durante el embarazo: $\chi^{2}(1,114)=6.205, p=.018$; $O R=3.394 \mathrm{y}$

r.- la presencia de pandillas juveniles en el barrio donde vivía el participante: $\chi^{2}(1$, $61)=4.638, p=.046 ; O R=5.283$.

Un porcentaje significativamente menor de varones con TD informaron no haber tenido relaciones sexuales, comparados con los adolescentes sin TD: $\chi^{2}(1,133)=5.323$, $p=.023 ; O R=.357$.

Un porcentaje mayor de mujeres con TD reportaron las siguientes situaciones comparadas con las mujeres sin TD:

a.- haber tenido relaciones sexuales con penetración: $\chi^{2}(1,36)=6.415, p=.027$; $O R=8.000$,

b.- no haber utilizado métodos anticonceptivos: $\chi^{2}(1,36)=6.841, p=.018 ; O R=9.583$; c.- pensamientos suicidas en los últimos 12 meses: $\chi^{2}(1,36)=4.946, p=.040$; $O R=6.111$,

d.- haber sido expulsada de algún plantel educativo por mal comportamiento: $\chi^{2}(1$, $36)=4.946, p=.040 ; O R=6.111$,

e.- haberse escapado de la casa por mala conducta personal: $\chi^{2}(1,36)=4.946, p=.040$; $O R=6.111$,

f.- por problemas con la ley: $\chi^{2}(1,36)=10.227$, $p=.006 ; O R=.636 \mathrm{y}$

g.- por las condiciones económicas de la familia: $\chi^{2}(1,36)=7.438, p=.023 ; O R=.727$.

Un porcentaje significativamente menor de mujeres con TD reportó estar estudiando, comparadas con sus pares: $\chi^{2}(1,36)=4.946$, $p=.040 ; O R=.164$.

Las comparaciones entre mujeres y varones con TD evidencian que ellas presentan un promedio significativamente mayor de:

a.- la edad que tenían la primera vez que fueron castigados físicamente de forma violenta [varones con TD: $M=8.04, D T=3.67$; $\mathrm{mu}-$ jeres con TD: $M=11.33, D T=2.00$; $t(34)=-3,322, p=.003 ; d=-1.11]$,

b.- puntuaciones en la Escala de Neuroticismo de la EPI (Eysenck, H.J. \& Eysenck, S.G.B., 1998) [varones con TD: $M=12.13$, $D T=4.96$; mujeres con TD: $M=15.91$,
$D T=3.78 ; t(71)=-2.888, p=.010 ;$ $d=-.857]$.

Las mujeres también presentaron una media más baja de puntuaciones en la Escala de Retroalimentación Personal de la CACIA (Capafóns \& Silva, 2001) [varones con TD: $M=9.98, D T=4.65$; mujeres con TD: $M=7.40, D T=2.76 ; t(34)=2.441, p=.025$; $d=.674]$.

Asimismo, informaron haber sido más frecuentemente castigadas físicamente de forma violenta que los varones con TD $[U(71)=173.0, p=.006]$ y un porcentaje mayor reportó las siguientes situaciones, comparados con dichos varones:

a.- pensamientos suicidas en los últimos 12 meses: $\chi^{2}(1,71)=4.623, p=.047 ; O R=.240$, b.- problemas en el trabajo por las conductas disociales informadas en el cuestionario diagnóstico: $\chi^{2}(1,71)=5.446, p=.034 ; O R=.212$.

Las adolescentes con TD, comparadas con los varones con TD, reportaron una frecuencia mayor de síntomas a nivel general y de los siguientes síntomas presentes en el cuestionario diagnóstico de Pineda y colaboradores (1999) -ver Tabla 2-:

a.-"Se ha quedado fuera de la casa en la noche sin permiso",

b.- "Engaña a otras personas para quitarles dinero u obtener objetos ajenos" y

c.- "Es cruel con las personas y le gusta hacerlas sufrir".

En todos estos casos, los tamaños del efecto oscilaron entre moderados y altos.

\section{Discusión}

El objetivo de la investigación realizada fue comparar un grupo de adolescentes varones y mujeres con TD entre sí y con respecto a un grupo de adolescentes varones y mujeres $\sin \mathrm{TD}$, para estudiar un conjunto de variables neuropsicológicas, biográficas, psicológicas / conductuales, familiares y comunitarias señaladas en la literatura especializada y los síntomas de dicho trastorno reportados por los participantes con TD.

Los resultados evidenciaron tanto similitudes como diferencias entre los participantes. 
Así, tanto los varones como las mujeres con TD, comparados con los varones y las mujeres sin dicho trastorno, respectivamente, presentaron un promedio mayor de puntuaciones en las escalas de Extroversión de la EPI (Eysenck, H.J. \& Eysenck, S.G.B., 1998), con tamaños del efecto moderados, de manera similar a lo encontrado en adolescentes varones por Daderman (1998), lo cual indica que este rasgo de personalidad podría incrementar el riesgo de TD. Asimismo, informaron una frecuencia mayor de consumo de marihuana (toda la vida y en los últimos 30 días), consumo de inhalantes, escapadas de casa, cambios de escuela, relaciones sexuales con penetración, pensamientos suicidas en los últimos 12 meses, haberse escapado de casa por mala conducta personal y por las condiciones económicas de la familia, haber sido expulsado(a) de un plantel educativo por mal comportamiento y una menor edad de inicio del consumo de marihuana.

Estos resultados tienden a confirmar que los adolescentes de ambos sexos con TD presentan dificultades similares que vulneran su calidad de vida y su salud física y mental, como el consumo de sustancias ilegales, conductas sexuales de riesgo, pensamientos suicidas, escapadas de casa y expulsiones escolares (Baker, 2008; Dicker, Morrissey, Abikoff \& Alvir, 1997; Fairchild et al., 2013; Harrington \& Maskey, 2008). En el caso de las adolescentes con TD, estos resultados son similares a los informados por Whitmore y colaboradores (1997), en relación con las conductas sexuales de riesgo y por Lehto-Salo y colaboradores (2009), en cuanto al riesgo suicida.

Si bien los varones con TD, comparados con sus homólogos, informaron un mayor número de parejas sexuales, las mujeres con TD, comparadas con las adolescentes sin dicho trastorno, reportaron haber comenzado su vida sexual a una edad menor y no utilizar preservativos, estos resultados señalan que tanto los varones como las mujeres con TD presentan más conductas sexuales de riesgo que los adolescentes sin dicho trastorno, aumentando la probabilidad de presentar embarazos no deseados (Fairchild et al., 2013; Keenan et al., 1999; Pager et al., 2007). De hecho, el riesgo de presentar relaciones sexuales con penetración y no utilizar preservativos fue ocho veces mayor entre estas adolescentes que las que no cumplían los criterios de TD.

Asimismo, los varones con TD comparados con sus homólogos, reportaron un mayor consumo de bebidas alcohólicas y cocaína, las mujeres con TD informaron un mayor consumo de éxtasis y bazuco, comparadas con sus homólogas, lo cual indica que ambos tienden a presentar un mayor consumo de sustancias psicoactivas (American Psychiatric Association, 2013; Harrington \& Maskey, 2008). Por lo tanto, la prevalencia de trastornos relacionados con sustancias podría ser similar entre los dos sexos y no mayor entre los varones, como encontraron Whitmore y colaboradores (1997) y Letho-Salo y colaboradores (2009), lo cual también es coherente con la mayor propensión al consumo en las adolescentes con TD resaltado recientemente por la American Psychiatric Association. Estos resultados, a su vez, tienden a ser coherentes con los del Estudio Nacional de Consumo de Sustancias Psicoactivas en Adolescentes en Conflicto con la Ley, en el que participaron 1.070 varones y 119 mujeres infractores institucionalizados entre 13 y 20 años, el cual halló que la prevalencia de consumo de alguna sustancia psicoactiva en el último año era significativamente mayor en estos jóvenes infractores, que en los jóvenes encuestados en una investigación realizada con población escolarizada. Por otra parte, aunque algunos de los motivos eran diferentes, tanto los varones como las mujeres con TD informaron haberse escapado de su casa con mayor frecuencia que sus homólogos, señalando en ambos casos la mala conducta personal como uno de los motivos con mayor riesgo.

A nivel psicológico / conductual, no obstante, se observaron diferencias importantes entre los varones y mujeres con TD que indican que las mujeres presentan más rasgos de personalidad que incrementan el riesgo de dicho trastorno, tal como informan las revisiones realizadas por Berkout y colaboradores (2011) y Keenan y colaboradores (1999). Así, las mujeres con TD, comparadas con sus ho- 
mólogas, presentaron una más baja empatía y capacidad de retroalimentación personal, una habilidad necesaria para presentar un comportamiento auto-controlado (Capafóns \& Silva, 2001), así como más rasgos de agresividad, dominación e intransigencia medidos con la Escala de Machismo (Lara, 1993), en todos los casos con tamaños del efecto elevados. Además, las adolescentes con TD presentaron mayores puntuaciones en Neuroticismo que los varones con dicho desorden y menores en Retroalimentación personal. Otra diferencia importante a nivel psicológico es la relacionada con el riesgo suicida, pues las mujeres con dicho trastorno reportaron una mayor frecuencia de pensamientos suicidas en los últimos 12 meses, comparadas con los varones con $\mathrm{TD}$, aunque con un riesgo más bajo $(O R=.240)$.

Es posible que estas diferencias a nivel de la personalidad sean las responsables de las discrepancias en las conductas disociales encontradas entre los varones y mujeres con $\mathrm{TD}$, que señalan, al contrario de lo resaltado por la American Psychiatric Association (2013) y lo encontrado en estudios previos, según Baker (2008), que las mujeres en esta población podrían presentar una frecuencia incluso mayor de conductas disociales tanto de tipo agresivo como de tipo furtivo, tales como quedarse fuera de casa, engañar para timar a otros, ser cruel y gustarle hacer sufrir a otras personas, lo cual es coherente con el promedio de puntuaciones significativamente mayor en rasgos de agresividad, dominación e intransigencia y los puntajes más bajos en empatía y autocontrol, encontrados en esta investigación y otras (Rey-Anacona, 2003; Taylor \& Iacono, 2007). Además, las mujeres con TD informaron más dificultades en el trabajo como consecuencia de dichas conductas disociales y fugas del hogar por problemas con la ley, aunque con un riesgo bajo.

A nivel neuropsicológico y biográfico, por otra parte, se encontró que las mujeres con TD, comparadas con las adolescentes sin dicho trastorno, presentaron menores puntuaciones en una prueba de fluidez fonémica, de manera similar a lo reportado por Haggerty y colaboradores (1998), lo que indica que este podría ser un factor de riesgo de TD también en las mujeres y no solo en los varones (Eme, 2007), quienes en este estudio evidenciaron puntuaciones más bajas en fluidez semántica. No obstante, las madres de dichas mujeres no reportaron más condiciones adversas durante su embarazo, lo cual sí ocurrió entre los adolescentes con TD comparados con los varones sin dicho trastorno, confirmando la idea de que dichas condiciones pueden ser un factor de riesgo biológico del TD (Harrington \& Maskey, 2008).

A nivel socio-familiar, por el contrario, se encontraron más circunstancias adversas entre los varones que entre las adolescentes con TD, que indican que estas circunstancias podrían incrementar más el riesgo de TD en ellos, como encontraron Lehto-Salo y colaboradores (2009). Así, los adolescentes con TD, comparados con sus homólogos, reportaron más frecuentemente haberse sentido descuidados por su padre, haber sido forzados sexualmente por una persona mayor, y sus madres informaron más frecuentemente pandillas juveniles en su lugar de vivienda, con un riesgo cinco veces mayor. Asimismo, comparados con las adolescentes con TD, dichos varones reportaron una edad menor en la que fueron castigados físicamente de forma violenta, aunque estas últimas informaron haber sido castigadas de dicha manera más frecuentemente que éstos.

Por otra parte, los resultados no son coherentes con los de algunos estudios citados en la Introducción, al no encontrarse ni entre los varones ni entre las mujeres diferencias en cuanto al temperamento en los primeros años de vida, la observación de violencia entre los padres o en medios masivos de comunicación, rasgos socio-demográficos como el número de hijos y el estado civil de los padres, antecedentes de criminalidad y consumo de alcohol y otros psicoactivos por parte de los padres, depresión materna y comorbilidad con el TDAH. Además, el hecho de que las madres de los adolescentes con TD presentaran puntuaciones significativamente más altas en dos de las cuatro subescalas de la Escala de Ajuste Diádico, que las de sus pares, señala que en su caso el funcionamiento ma- 
rital no necesariamente incrementa el riesgo de presentar TD. Estas discrepancias con respecto a estudios previos señalan que en esta población de adolescentes, los factores psicológicos como los evidenciados en este estudio, tendrían un peso mayor en la predisposición a dicho trastorno que los factores socio-familiares, los cuales tendrían un peso mayor en los contextos socioculturales en los que se llevaron a cabo dichos estudios.

En conclusión, los resultados obtenidos señalan que: (a) las adolescentes con TD pueden presentar una frecuencia mayor de conductas disociales y cuadros de conducta antisocial similares o más graves que la de los adolescentes con TD, (b) los dos presentan una alta frecuencia de condiciones y conductas que vulneran su salud física y mental, lo que puede empeorar su pronóstico y (c) los rasgos de personalidad parecen asociarse más con el TD en las mujeres, y las características socio-familiares más en los varones, ya que las mujeres con TD de este estudio presentaron puntuaciones más bajas en empatía y retroalimentación personal, comparadas con las adolescentes sin TD, así como puntuaciones más altas en Neuroticismo y un mayor riesgo suicida que los varones con $\mathrm{TD}$, mientras que estos últimos informaron más frecuentemente sentirse descuidados por sus padres, haber sido forzados sexualmente y sus progenitoras reportaron con mayor frecuencia pandillas juveniles en el sector de vivienda de sus hijos.

Por consiguiente, en la evaluación y tratamiento de casos con TD deberían considerarse estas diferencias, de manera que en el caso de las mujeres se haga mayor énfasis en el tratamiento de habilidades y aspectos socio-emocionales como la empatía y la regulación emocional, sin desconsiderar las variables socio-familiares, mientras que en el caso de los varones se debería brindar más importancia a estas circunstancias, sin descuidar los aspectos individuales. Asimismo, es importante destacar que los resultados de esta investigación indican que los rasgos de índole neuropsicológico, como la baja fluidez verbal, serían también característicos de las adolescentes con TD y por consiguiente deberían considerarse en su evaluación y tratamiento. Igualmente, dichos resultados señalan que se debería brindar una importancia mayor a la prevención terciaria en el abordaje de casos de adolescentes con TD, con alternativas de tratamiento que minimicen el efecto de las múltiples conductas de riesgo para la salud física y mental que evidencian estos adolescentes, como el consumo de bebidas alcohólicas y otras sustancias psicoactivas, las conductas sexuales de riesgo, pensamientos suicidas y fugas del hogar, entre otras.

Se puede considerar que las principales fortalezas del estudio realizado fueron el amplio número de variables examinadas, así como la utilización de una muestra de comparación similar a la de los varones y mujeres con TD. Sin embargo, este estudio presenta como principales debilidades el tamaño de las muestras, particularmente el número de mujeres que era muy bajo, así como el nivel socioeconómico de los participantes, puesto que la mayoría vivía en barrios de estratos bajos, lo cual limita la generalización de los resultados a adolescentes con TD institucionalizados de dichos estratos. Asimismo, el número de participantes varones con TD era superior al de mujeres con dicho trastorno y no se hizo una evaluación del coeficiente intelectual de los participantes, lo que hubiera permitido descartar que dicha variable pudiera ser la responsable de las diferencias que se observaron en fluidez fonémica entre los dos grupos de mujeres, aspectos todos que deberán ser subsanados en futuras investigaciones similares.

En particular, se recomienda que en futuros estudios se evalúe el efecto diferencial por sexo de aquellas alternativas de tratamiento del TD que no han sido objeto de esta evaluación, como así también que se diseñen y prueben empíricamente programas de tratamiento que sean sensibles a dichas diferencias, que se profundice en las características neurobiológicas de las adolescentes con TD, tal como lo señalan Fairchild y colaboradores (2013) y que se incluyan en el tratamiento de los adolescentes de ambos sexos con dicho trastorno, estrategias para disminuir sus secuelas sobre la salud física y psicológica. 
Diferencias entre adolescentes con TD y sin TD

TABLA 1

VARIABLES ESTUDIADAS E INSTRUMENTOS ADMINISTRADOS

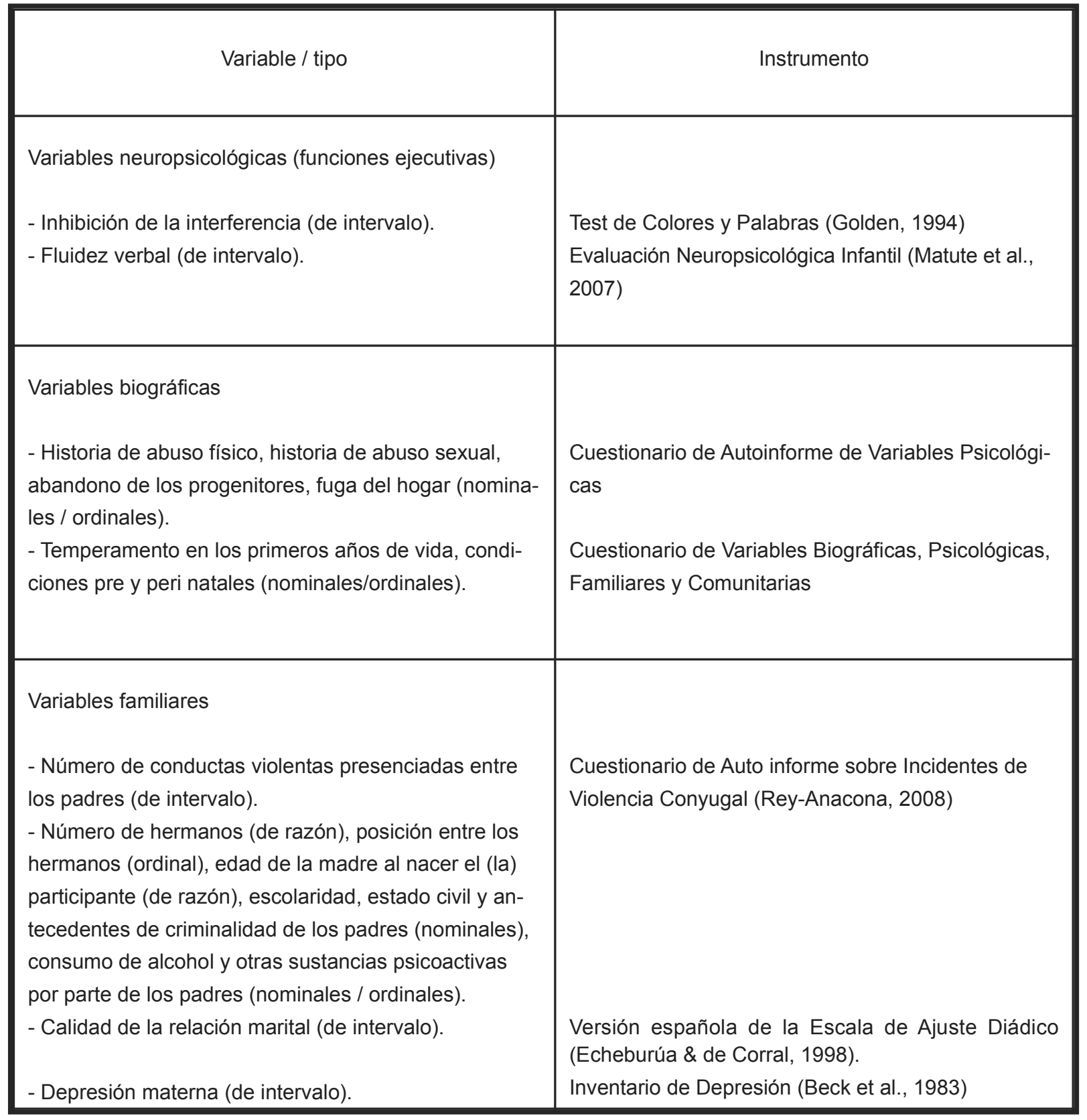

Continúa 
TABLA 1(CONTINUACIÓN)

VARIABLES ESTUDIADAS E INSTRUMENTOS ADMINISTRADOS

\begin{tabular}{|c|c|}
\hline Variable / tipo & Instrumento \\
\hline $\begin{array}{l}\text { Variables psicológicas / conductuales } \\
\text { - Consumo de alcohol y otros psicoactivos, conducta se- } \\
\text { xual de riesgo, ideación suicida, intentos de suicido, rela- } \\
\text { ción con pares y adultos, relación con padres y herma- } \\
\text { nos, rendimiento académico, repetición de años escolares, } \\
\text { observación de programas de televisión con contenido vio- } \\
\text { lento (nominales / ordinales). } \\
\text { - Rasgos de personalidad de agresividad, dominación e in- } \\
\text { transigencia (de intervalo). } \\
\text { - Empatía (de intervalo). } \\
\text { - Extroversión y estabilidad emocional (de intervalo). } \\
\text { - Autocontrol (de intervalo). } \\
\text { - Cumplimiento de criterios diagnósticos del TDAH a nivel } \\
\text { general y por subtipo (nominales). }\end{array}$ & $\begin{array}{l}\text { Cuestionario de Autoinforme de Variables Psicoló- } \\
\text { gicas } \\
\text { IMAFE (Lara, 1993) } \\
\text { Escala de Empatía (Rey-Anacona, 2003) } \\
\text { EPI (Eysenck, H.J. \& Eysenck, S.G.B., 1984) } \\
\text { CACIA (Capafóns \& Silva, 2001) } \\
\text { Cuestionario Breve para el Diagnóstico de Defi- } \\
\text { ciencia Atencional (Pineda et al., 1999) }\end{array}$ \\
\hline $\begin{array}{l}\text { Variables comunitarias } \\
\text { - Servicios comunitarios de la familia y acceso a ellos, pe- } \\
\text { ligrosidad del sector de vivienda, presencia de pandillas } \\
\text { juveniles (nominales / ordinales). }\end{array}$ & $\begin{array}{l}\text { Cuestionario de Variables Biográficas, Psicológicas, } \\
\text { Familiares y Comunitarias }\end{array}$ \\
\hline
\end{tabular}

Nota:

Las variables categorizadas como "Nominales / ordinales" fueron analizadas con base en información de estos dos niveles de medición. 
TABLA 2

Diferencias EN LA FRECUENCIA DE SÍNTOMAS DE TD

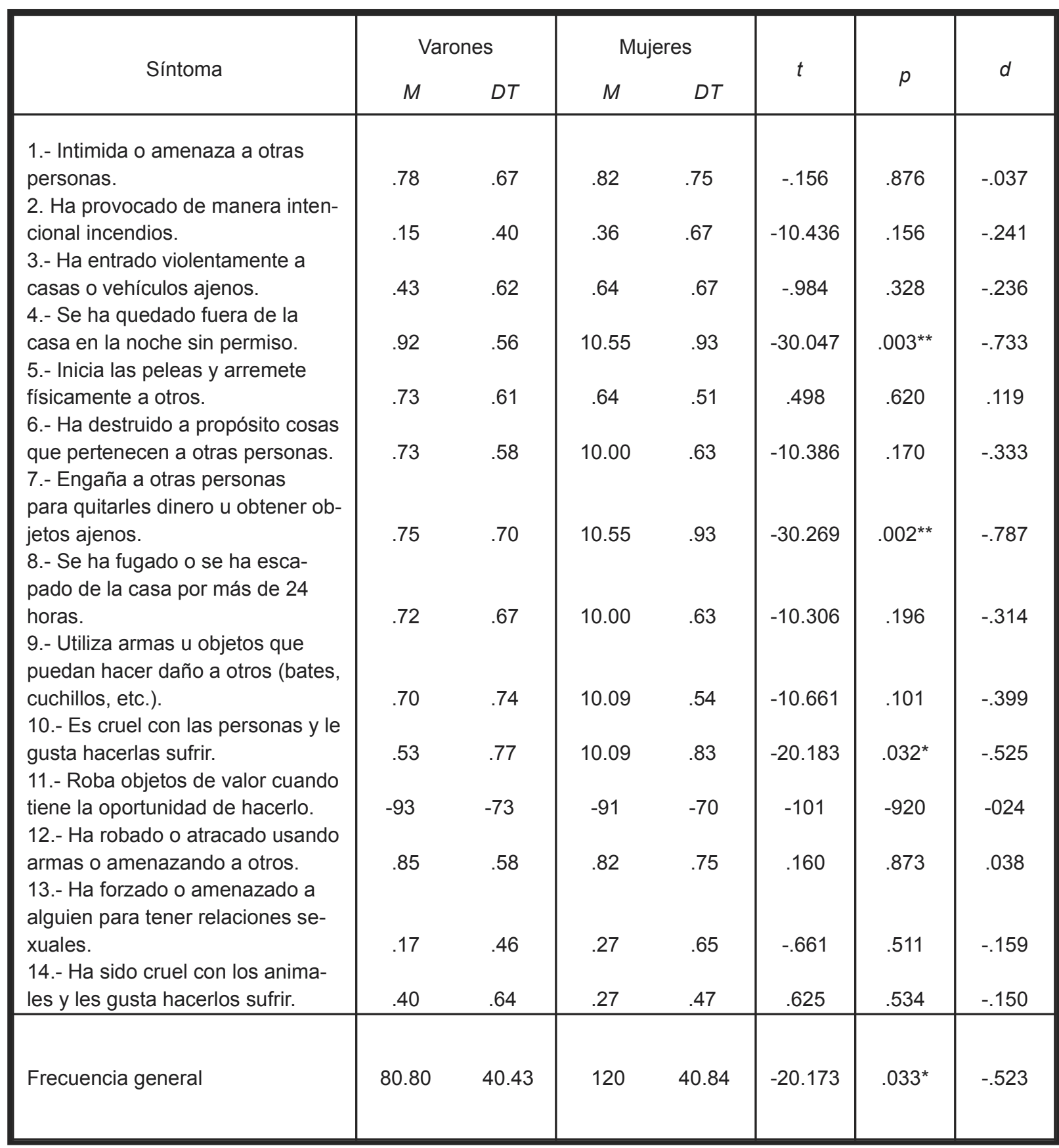

$g l=69$

${ }^{*} p \leq .05$, dos colas

${ }^{* *} p \leq .01$, dos colas 


\section{REFERENCIAS BIBLIOGRÁFICAS}

American Psychiatric Association. (2013). Diagnostic and Statistical Manual of Mental Disorders (5a. ed.). Arlington, VA: Autor.

Baker, K. (2008). Conduct disorders in children and adolescents. Paediatrics and Child Health, 19(2), 73-78. http://dx.doi.org/10.1016/j. paed. 2008.10.008

Beck, A., Rush, A., Shaw, B. \& Emery, G. (1983). Terapia cognitiva de la depresión [Cognitive therapy of depression]. Bilbao: Desclée de Brouwer.

Berkout, O.V., Young, J.N. \& Gross, A.M. (2011). Mean girls and bad boys: Recent research on gender differences in conduct disorder. $A g-$ gression \& Violent Behavior, 16(6), 503-511. http://dx.doi.org/10.1016/j.avb.2011.06. 001

Brener, N.D., Kann, L., Kinchen, S.A. et al. (2004). Methodology of the Youth Risk Behavior Surveillance System. Morbidity \& Mortality Weekly Report, 53 (RR-12), 1-13.

Capafóns, A. \& Silva, F. (2001). Cuestionario de Autocontrol Infantil y Adolescente [Child and adolescent self-control questionnaire] (5ta. ed. revisada). Madrid: TEA.

Daderman, A.M. (1998). Differences between severely conduct-disordered juvenile males and normal juvenile males: The study of personality traits. Personality and Individual Differences, 26(5), 827-845. http://dx.doi.org/10. 1016/S0191-8869(98)00186-X

Dicker, R., Morrissey, R., Abikoff, H. \& Alvir, J. (1997). Hospitalizing the suicidal adolescent: Decision-making criteria of psychiatric residents. Journal of the American Academy of Child and Adolescent Psychiatry, 36(6), 769776. http://dx.doi.org/10.1097/00004583-199 706000-00013

Echeburúa, E. \& de Corral, P. (1998). Manual de violencia familiar [Handbook of family violence]. Madrid: Siglo Veintiuno.
Eme, R.F. (2007). Sex differences in child-onset, life-course-persistent conduct disorder. A review of biological influences. Clinical Psychology Review, 27, 607-627. http://dx.doi.org/ 10.1016/j.cpr.2007.02.001

Eme, R.F. \& Kavanauhg, L. (1995). Sex differences in conduct disorder. Journal of Clinical Child Psychology, 24(4) 406- 426.

Evans, S.E., Davis, C. \& DiLillo, D. (2008). Exposure to domestic violence: A meta-analysis of child and adolescent outcomes. Aggression and Violent Behavior, 13(2), 131-140. http://dx.doi. org/10.1016/j.avb. 2008.02. 005

Eysenck, H.J. \& Eysenck, S.G.B. (1998). Cuestionario de Personalidad de Eysenck [Eysenck Personality Inventory] (8a. ed.). Madrid: TEA.

Fairchild, G., Hagan, C.C., Walsh, N.D., Passamonti, L., Calder, A.J. \& Goodyer, I.M. (2013). Brain structure abnormalities in adolescent girls with conduct disorder. Journal of Child Psychology and Psychiatry, 54(1), 86-95. http://dx.doi.org/10.1111/j.1469-7610.2012.02 617.x

Fairchild, G., Van Goozen, S.H.M., Stollery, S.J., Brown, J., Gardiner, J., Herbert, J. \& Goodyer, I.M. (2008). Cortisol diurnal rhythm and stress reactivity in male adolescents with early-onset or adolescence-onset conduct disorder. Biological Psychiatry, 64(7), 559-606. http://dx.doi. org/10.1016/ j.biopsych.2008. 05. 022

Frick, P.J., Christian, R.E. \& Wooton, J.M. (1999). Age trends in association between parenting practices and conduct problems. Behavior Modification, 23(1), 106-128. http://dx.doi.org/ 10.1177/0145445599231005

Golden, C.J. (1994). Test de Colores y Palabras [Colours and Words Test]. Madrid: TEA.

Haggerty, J.C., Nevid, J.S. \& Moulton, J.L. (1998). Anxiety and cognitive performance in adolescent women with disruptive behavior disorders. Journal of Clinical Psychology, 54 (8), 1017-1027. 
Harrington, R. \& Maskey, S. (2008). Behaviour disorders in children and adolescents. Medicine, 36(9), 482-485. http://dx.doi.org/10.101 6/j.mpmed.2008.06.010

Ilomäki, E., Viilo, K., Hakko, H., Marttunen, M., Mäkikyrö, T., Räsänen, P. \& STUDY-70 workgroup (2006). Familial risks, conduct disorder and violence: A finnish study of 278 adolescent boys and girls. European Child \& Adolescent Psychiatry, 15(1), 46-51. http://dx.doi.org/10. 1007/s00787-006-0507-x

Keenan, K., Loeber, R. \& Green, S. (1999). Conduct disorder in girls: A review of the literature. Clinical Child Family Psychology, 2 (1), 3-19.

Lara, M.A. (1993). Inventario de Masculinidad y Femineidad [Inventory of Masculinity and Femininity]. México: El Manual Moderno.

Lehto-Salo, P., Närhi., V., Ahonen, T. \& Marttunen, M. (2009). Psychiatric comorbidity more common among adolescent females with CD/ODD than among males. Nordic Journal of Psychiatry, 63(4), 308-315. http://dx.doi. org/10.1080/08039480902730615

Lewis, C.F. (2010). Childhood antecedents of adult violent offending in a group of female felons. Behavior Sciences and the Law, 28(2), 224-234. http://dx.doi.org/10.1002/bsl.929

Marino, J., Acosta Mesas, A. \& Zorza, J.P. (2011). Control ejecutivo y fluidez verbal en población infantil: Medidas cuantitativas, cualitativas y temporales [Executive control and verbal fluency in child population: Quantitative, qualitative and temporal measures]. Interdisciplinaria, 28 (2), 245-260.

Masi, G., Milone, A., Manfredi, A., Pari, C., Paziente, A. \& Millepiedi, S. (2007). Conduct disorder in referred children and adolescents: Clinical and therapeutic issues. Comprehensive Psychiatry, 49(2), 146-153. http://dx.doi.org/1 0.1016/j.comppsych.2007.08.009

Martínez-Gómez, J.A., Guerrero-Rodríguez, S.I. \& Rey-Anacona, C.A. (2012). Evaluación de la validez de constructo y la confiabilidad del Inventario de Masculinidad y Femineidad en adolescentes y adultos jóvenes colombianos [Assessment of construct validity and reliability of the Inventory of Masculinity and Femininity in adolescents and young Colombian adults]. Avances en Psicología Latinoamericana, 30(1), 170-181.

Martorell, M.C., González, R. \& Calvo, A. (1998). Cuestionario de Conducta Prosocial [Prosocial Behavior Questionnaire]. Manuscrito no publicado, Universidad de Valencia.

Matute, E., Roselli, M., Ardila, A. \& OstroskySolís, F. (2007). Evaluación neuropsicológica infantil [Child neuropsychological assessment]. México: Manual Moderno.

Moeller-Nehring, E., Moach, A., Castell, R., Weigel, A. \& Meyer, M. (1998). Conditions facilitating social disorder in a clinic-referred sample. Praxis der Kinderpsychologie und Kinderpsychiatrie, 47(1), 36-47.

Ohannessian, C.M., Hesselbrock, V.M., Kramer, J., Kuperman, S., Bucholz, K.K., Schuckit, M.A. \& Nurnberger, J.I. (2005). The relationship between parental psychopathology and adolescent psychopathology: An examination of gender patterns. Journal of Emotional and Behavioral Disorders, 13(2), 67-76. http://dx. doi.org/ 10.1177/10634266050130020101

Pager, K., Kazmi, A., Gardner, W.P. \& Wang, Y. (2007). Female conduct disorder: Health status in young adulthood. Journal of Adolescent Health, 40(1), 84.e1- 84.e7. http://dx.doi.org/ 10.1016/j.jadohealth.2006.07.012

Pager, K., Leininger, L. \& Gardner, W. (2010). Recognition of facial affect in girls with conduct disorder. Psychiatry Research, 175(3), 244-251. http://dx.doi.org/10. 1016/j.psychres. 2009.06.003

Pager, K., Tabbah, R., Gardner, W., Rubin, R.T., Czambell, R.K. \& Wang, Y. (2006). Adrenal androgen and gonadal hormone adolescent girls 
with conduct disorder. Psychoneuroendocrinology, 31, 1245-1256. http://dx.doi.org/10.10 16/j.psyneuen.2006.09.005

Pineda, D.A. (2000). La función ejecutiva y sus trastornos [Executive function and its disorders]. Revista de Neurología, 30(8), 764-768.

Pineda, D.A., Henao, G.C., Puerta, I.C., Mejía, S.E., Gómez, L.F., Miranda, M.L., Roselli, M., Ardila, A., Restrepo, M.A., Murelle, L. \& Grupo de Investigación de la Fundación Universidad de Manizales (1999). Uso de un cuestionario breve para el diagnóstico de deficiencias de atención [The usefulness of a short questionnaire for the diagnosis of attention deficiency]. Revista de Neurología, 28(4), 365372.

Pineda, D.A., Puerta, I.C., Arango, C.P., Calad, O.M. \& Villa, M.T. (2000). Cuestionario Breve para el Diagnóstico del Trastorno Disocial de la Conducta en Adolescentes de 12 a 16 Años [A brief questionnaire for diagnosis of maladaptation behaviour disorders in adolescents aged between 12 and 16 years]. Revista de Neurología, 30(12), 1145-1150.

Posada-Villa, J.A., Aguilar-Gaxiola, S.A., Magaña, C.G. \& Gómez, L.C. (2004). Prevalencia de trastornos mentales y uso de servicios: Resultados preliminares del Estudio Nacional de Salud Mental. Colombia, 2003 [Prevalence of mental disorders and use of services: Preliminary results National Mental Health Study. Colombia, 2003]. Revista Colombiana de Psiquiatría, 33(3), 241-262.

Raine, A., Yang, Y., Narr, K.L. \& Toga, A.W. (2011). Sex differences in orbitofrontal gray as a partial explanation for sex differences in antisocial personality. Molecular Psychiatry, 16, 227-236. http://dx.doi.org/10.1038/mp.2009.1 36

Renner, L.M. \& Slack, K.S. (2006). Intimate partner violence and child maltreatment: Understanding intra- and intergenerational connec- tions. Child Abuse \& Neglect, 30(6), 599-617. http://dx.doi.org/10.1016/j.chiabu. 2005.12.005 Rey-Anacona, C.A. (2003). Adaptación y validación de una escala para medir empatía en preadolescentes y adolescentes varones [Adaptation and validation of a scale to measure empathy in preadolescent and adolescent boys]. Revista Latinoamericana de Psicología, 34(2), 185194.

Rey-Anacona, C.A. (2008). Habilidades pro sociales, rasgos de personalidad de género y aceptación de la violencia hacia la mujer, en adolescentes que han presenciado violencia entre sus padres [Pro-social skills, gender personality traits and acceptance of violence toward women in adolescents who have witnessed violence in their parents]. Acta Colombiana de Psicología, 11(1), 107-118.

Rioseco, P., Vicente, B., Saldivia, S., Cova, F., Melipillán, R. \& Rubi, P. (2009). Prevalencia de trastornos psiquiátricos en adolescentes infractores de ley. Estudio caso-control [Prevalence of psychiatric disorder among juvenile offenders. Case-control study]. Revista Chilena de Neuropsiquiatría, 47(3), 190-200. http://dx. doi.org/10.4067/S0717-92272009000300003

Rutter, M., Giller, H. \& Hagell, A. (2000). La conducta antisocial de los jóvenes [Antisocial behavior by young people]. Madrid: Cambridge University Press.

Schmeck, K. \& Poutstka, F. (2001). Temperament and disruptive behavior disorders. Psychopathology, 34(3), 159-163. http://dx.doi.org/ $10.1159 / 000049300$

Spanier, G.B. (1976). Measuring dyadic adjustment: New scales for assessment the quality of marriage and similar dyads. Journal of Marriage and Family, 38(1), 15-28.

Taylor, J. \& Iacono, W.G. (2007). Personality trait differences in boys and girls with clinical or sub-clinical diagnoses of conduct disorder versus antisocial personality disorder. Journal of 
Adolescence, 30(4), 537-547. http://dx.doi.or g/10.1016/j.adolescence.2006.09.003

Toupin, J., Dery, M., Pauze, R., Mercier, H. \& Fortin, L. (2003). Cognitive and familial contributions to conduct disorder in children. Journal of Child Psychology and Psychiatry, 41(3), 333-344. http://dx.doi.org/10.1111/146 9-7610.00617
Whitmore, E.A., Mikulich, S.K., Thompson, L.L., Riggs, P.D., Aarons, G.A. \& Crowley, T.J. (1997). Influences on adolescent substance dependence: Conduct disorder, depression, attention deficit hyperactivity disorder, and gender. Drug and Alcohol Dependence, 47(2), 87-97.

Grupo de Investigación en Psicología Clínica y de la Salud Universidad Pedagógica y Tecnológica de Colombia Tunja - Colombia

Fecha de recepción: 31 de diciembre de 2013 Fecha de aceptación: 17 de junio de 2014 
